\title{
A tightly coupled rotational SINS/CNS integrated navigation method for aircraft
}

\author{
NING Xiaolin, YUAN Weiping, and LIU Yanhong* \\ School of Instrument and Optoelectronic Engineering, Beihang University, Beijing 100191, China
}

\begin{abstract}
Strapdown inertial navigation system (SINS)/celestial navigation system (CNS) integrated navigation is widely used to achieve long-time and high-precision autonomous navigation for aircraft. In general, SINS/CNS integrated navigation can be divided into two integrated modes: loosely coupled integrated navigation and tightly coupled integrated navigation. Because the loosely coupled SINS/CNS integrated system is only available in the condition of at least three stars, the latter one is becoming a research hotspot. One major challenge of SINS/CNS integrated navigation is obtaining a high-precision horizon reference. To solve this problem, an innovative tightly coupled rotational SINS/CNS integrated navigation method is proposed. In this method, the rotational SINS error equation in the navigation frame is used as the state model, and the starlight vector and star altitude are used as measurements. Semi-physical simulations are conducted to test the performance of this integrated method. Results show that this tightly coupled rotational SINS/CNS method has the best navigation accuracy compared with SINS, rotational SINS, and traditional tightly coupled SINS/CNS integrated navigation method.
\end{abstract}

Keywords: celestial navigation system (CNS), rotation modulation technology, rotational strapdown inertial navigation system (SINS), rotational SINS/CNS integrated navigation.

DOI: $10.21629 / J S E E .2019 .04 .14$

\section{Introduction}

For aircraft guidance, the strapdown inertial navigation system (SINS) is promising. It is completely autonomous and has a high output rate, but its usage as a standalone navigation system is limited because the navigation errors increase with time [1]. A celestial navigation system (CNS) can provide both attitude and position information. The navigation accuracy of CNS has nothing to do with time and distance, but rather with the accuracy of measurements, especially the precision of the horizon reference [2]. Considering the complementary characteristics of SINS

\footnotetext{
Manuscript received August 13, 2018.

*Corresponding author.

This work was supported by the National Natural Science Foundation of China (61722301).
}

and CNS, the SINS/CNS integrated navigation is a suitable choice for aircraft, which can achieve autonomous and high-accuracy navigation.

Until now, the loosely coupled integrated method [36] and the tightly coupled integrated method [7,8] have been investigated briefly. In the loosely coupled integrated method, the position and attitude of the aircraft are calculated by the CNS and INS independently, and then the difference between the position or attitude obtained through CNS and through SINS is used as measurement. Compared with these two navigation methods alone, the accuracy of this integrated method is greatly improved. However, the precondition for this integrated method is that the CNS must simultaneously observe three or more stars through the star sensor with a large field-of-view (FOV) to obtain the aircraft's attitude matrix. A tightly coupled integrated method $[7,8]$ provides a new framework for SINS/CNS integration, which enables direct fusion of SINS measurements with CNS observations, such as the star vector and the star altitude obtained by observing only one star. The benefits of this integrated method lie in two aspects. Firstly, this integrated method only needs to observe one star, which is an overwhelming advantage over the loosely coupled integrated method. Secondly, since the original star observations are directly input into the tightly coupled integrated method fusion filter, the stochastic models avoid being used twice. As a result, the navigation accuracy is improved. Since the tightly coupled SINS/CNS integrated navigation has a wider range of applications and higher navigation accuracy, it is becoming a hotspot for future research.

Ning et al. [7] proposed a two-mode tightly coupled SINS/CNS navigation method. When the rover traverses, the starlight is used as measurement. When the rover is at rest, the starlight vector, star altitude, and horizontal velocity errors are used as measurements. The twomode method can not only restrict the attitude error, but also restrain the position error in a limited range com- 
pared with traditional odometry. Wang et al. [8] proposed a framework for INS/CNS integrated navigation, which enables to directly fuse the INS measurement information with information from the original CNS observation. The difference of star altitude provided by the INS and the CNS is used as measurements, and an adaptive integrated algorithm with weighted multi-star observations is implemented to fuse the INS and CNS data. In a disturbance environment, this method shows stronger navigation stability and higher navigation accuracy than the classic INS/CNS integrated navigation.

For spacecraft, the stellar refraction method can directly obtain the Earth's horizon reference [9]. Nevertheless, this method is not applicable for aircraft. Since in the case of the horizon reference can be only provided by the SINS for aircraft, an improvement of the SINS accuracy is of paramount importance. There are two kinds of methods to improve the accuracy of SINS, one is to improve the accuracy of inertial sensors, and the other is to use error compensation technology. In this study, the rotation modulation technique, a kind of error compensation technology, is used to improve the navigation performance.

Rotating inertial navigation has been developed for many years [10,11]. The inertial measurement unit (IMU) is mounted on a rotary table. The rotary table rotates the IMU back and forth to modulate the bias errors of the gyros and accelerometers. By using the rotation modulation technique, the gyro drifts and acceleration biases can be automatically compensated [12]. The rotation scheme of the rotational SINS will directly affect the system's structure and the costs. Further, it will affect the navigation accuracy [13]. An improper rotation scheme will increase the navigation errors and deteriorate the system's performances, so the rotation scheme should be carefully designed. The rotation scheme for a single-axis is applied, and the IMU periodically rotates along a single rotation axis. In this paper, the single-axis reciprocating rotation is used to modulate the bias errors of inertial sensors. Because the horizon reference provided by the rotational SINS is more precise than that of the SINS, an innovative method of a tightly coupled rotational SINS/CNS integrated navigation is proposed.

This paper is divided into five sections. Following this introduction, the principle of the rotational SINS and $\mathrm{CNS}$, and the rotation scheme analysis of the rotational SINS are outlined in Section 2. Then, the state model and the measurement model of the tightly coupled rotational SINS/CNS integrated navigation are described in Section 3. Semi-physical simulations of the tightly coupled rotational SINS/CNS integrated navigation with some comparisons are presented in Section 4 to demonstrate the performance improvement. In addition, the impact of the rotation scheme, the gyros' accuracy, the accelerometers' accuracy, the star sensor's accuracy, IMU installation errors, and star sensor installation errors in the navigation performance of the tightly coupled rotational SINS/CNS integrated navigation are analyzed. In Section 5, a brief conclusion is drawn.

\section{Principle of the rotational SINS and CNS}

Many coordinate frames shown in Fig. 1 will be used in this paper, including the earth-centered inertial frame (i-frame), the earth-fixed frame (e-frame), the geography frame taken as the navigation frame (n-frame), the aircraft body frame (b-frame), the IMU coordinate frame ( $p$ frame), and the star sensor frame (s-frame) [14].

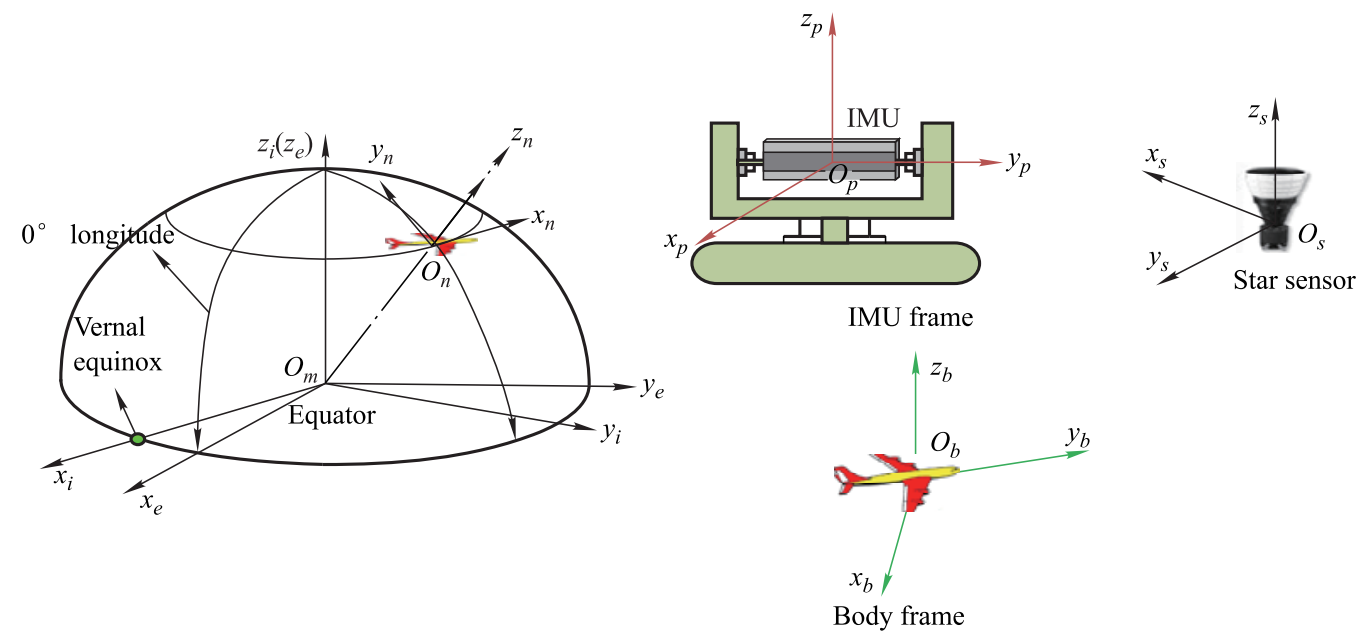

Fig. 1 Reference frames 


\subsection{Principle of the rotational SINS}

The rotational SINS mainly consists of the inertial measurement unit (IMU), the inertial navigation mechanization, and the rotary table. The IMU is composed of three orthogonal accelerometers and three orthogonal gyroscopes, which are used to measure the angular rate $\boldsymbol{w}_{i p}^{p}$ and the specific force $\boldsymbol{f}_{i p}^{p}$ of the IMU. The IMU is mounted on the rotator assembly, which is used to measure the rotation angle and remove the navigation errors periodically introduced by drifts of the inertial sensors. With the initial information, the measurements can be transformed to $\mathrm{n}$-frame to obtain velocity, position, and attitude of the aircraft [15]. The equation of the rotational SINS in the n-

$$
\boldsymbol{C}_{n}^{b}=\left(\boldsymbol{C}_{b}^{n}\right)^{\mathrm{T}}=\left[\begin{array}{c}
\cos \theta \cos \psi-\sin \theta \sin \varphi \sin \psi \\
\cos \theta \sin \psi+\sin \theta \sin \varphi \cos \psi \\
-\sin \theta \cos \varphi
\end{array}\right.
$$

where $\theta, \varphi, \psi$ are pitch, roll and yaw angles, respectively. $C_{b}^{p}$ is determined by the rotation angle. The rotational SINS is a single-axis system with the $z_{b}$ axis, and the rotation rate of the rotary table is denoted by $w . C_{b}^{p}$ can be denoted [17] as

$$
\boldsymbol{C}_{b}^{p}=\left(\boldsymbol{C}_{p}^{b}\right)^{\mathrm{T}}=\left[\begin{array}{ccc}
\cos (w t) & \sin (w t) & 0 \\
-\sin (w t) & \cos (w t) & 0 \\
0 & 0 & 1
\end{array}\right]
$$

where $t$ is the time interval. $\boldsymbol{v}_{n}=\left[v_{E}^{n}, v_{N}^{n}, v_{U}^{n}\right]^{\mathrm{T}}$ is the aircraft's velocity expressed in the n-frame, $\boldsymbol{r}_{n}=$ $[L, \lambda, h]^{\mathrm{T}}$ denotes the aircraft's position, $L$ is the latitude, $\lambda$ is the longitude, and $h$ is the altitude, $f_{i p}^{p}$ is the specific force measured by the accelerometer, $\boldsymbol{g}_{n}$ is the gravity acceleration expressed in the n-frame, $\boldsymbol{\omega}_{i e}^{n}=$ $\left[0, \omega_{i e} \cos L, \omega_{i e} \sin L\right]^{\mathrm{T}}$ is the earth's rotation rate denoted in the n-frame, and $\omega_{i e}$ has a value of $7.2921 \times 10^{-5} \mathrm{rad} / \mathrm{s}$, $\boldsymbol{\omega}_{e n}^{n}=\left[-v_{N}^{n} / R_{y}, v_{E}^{n} / R_{x}, v_{E}^{n} \tan L / R_{x}\right]^{\mathrm{T}}$ is the angular velocity of the $\mathrm{n}$-frame relative to the e-frame expressed in the $\mathrm{n}$-frame, $R_{x}$ and $R_{y}$ are the prime vertical radius and the meridian circle radius, respectively, $\boldsymbol{\Omega}_{n p}^{p}$ is a skew symmetric matrix of $\boldsymbol{\omega}_{n p}^{p}, \boldsymbol{\omega}_{n p}^{p}=\left[\omega_{n p x}^{p}, \omega_{n p y}^{p}, \omega_{n p z}^{p}\right]^{\mathrm{T}}$ is the angular velocity of the $\mathrm{p}$-frame relative to the $\mathrm{n}$-frame expressed in the $\mathrm{p}$-frame, which is given by

$$
\boldsymbol{\omega}_{n p}^{p}=\boldsymbol{\omega}_{i p}^{p}-\boldsymbol{C}_{n}^{p} \boldsymbol{\omega}_{i n}^{n}=\boldsymbol{\omega}_{i p}^{p}-\boldsymbol{C}_{n}^{p}\left(\boldsymbol{\omega}_{i e}^{n}+\boldsymbol{\omega}_{e n}^{n}\right)
$$

where $\omega_{i p}^{p}$ denotes the gyroscope output.

\subsection{Rotation scheme analysis of the rotational SINS}

The rotation scheme is one main content of the rotation modulation technology [18]. The rotation scheme in-

$$
\boldsymbol{C}_{n}^{b}=\left(\boldsymbol{C}_{b}^{n}\right)^{\mathrm{T}}=\left[\begin{array}{c}
\cos \theta \cos \psi-\sin \theta \sin \varphi \sin \psi \\
\cos \theta \sin \psi+\sin \theta \sin \varphi \cos \psi \\
-\sin \theta \cos \varphi
\end{array}\right.
$$

frame [16] can be written as

$$
\begin{gathered}
\left\{\begin{array}{l}
\dot{\boldsymbol{C}}_{n}^{p}=-\boldsymbol{\Omega}_{n p}^{p} \boldsymbol{C}_{n}^{p} \\
\dot{\boldsymbol{v}}_{n}=\boldsymbol{C}_{p}^{n} \boldsymbol{f}_{i p}^{p}-\left(2 \boldsymbol{\omega}_{i e}^{n}+\boldsymbol{\omega}_{e n}^{n}\right) \times \boldsymbol{v}_{n}+\boldsymbol{g}_{n} \\
\dot{\boldsymbol{r}}_{n}=\boldsymbol{C} \boldsymbol{v}_{n}
\end{array}\right. \\
\boldsymbol{C}=\left[\begin{array}{ccc}
0 & 1 / R_{y} & 0 \\
1 /\left(R_{x} \cos L\right) & 0 & 0 \\
0 & 0 & 1
\end{array}\right]
\end{gathered}
$$

where $C_{n}^{p}$ is the attitude matrix of the IMU and can be obtained by the product of the aircraft's attitude matrix $C_{n}^{b}$ and the rotation matrix $\boldsymbol{C}_{b}^{p}$. This $\boldsymbol{C}_{n}^{b}$ can be defined using rotations sequence of 2-1-3 as

$$
\left.\begin{array}{cc}
-\cos \varphi \sin \psi & \sin \theta \cos \psi+\cos \theta \sin \varphi \sin \psi \\
\cos \varphi \cos \psi & \sin \theta \sin \varphi-\cos \theta \sin \varphi \cos \psi \\
\sin \varphi & \cos \theta \cos \varphi
\end{array}\right]
$$

cludes a single-axis unidirectional and reciprocating rotation, a single-axis rotation with four angle positions $\left(-135^{\circ},+45^{\circ},+135^{\circ},-45^{\circ}\right)$, a dual-axis alternate rotation, and a dual-axis continuous rotation [19]. In this paper, the single-axis reciprocating rotation is taken as an example, and the rotation scheme is the relationship between the rotational axis $z_{b}$ and the sensing axis $z_{p}$, as shown in Fig. 2, where $\theta_{b p}$ is the installation angle between the $z_{b}$ axis and the $z_{p}$ axis.

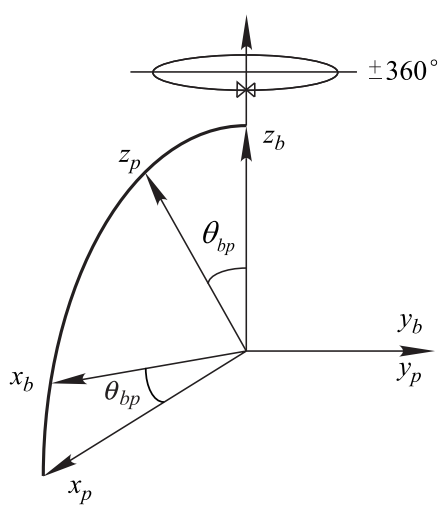

Fig. 2 Installation angle between the IMU and the rotary table

The effect of rotation modulation on accelerometer biases is the same as that on gyro drifts. Thus, only the effect on the gyro drifts are analyzed [20]. The principle of the rotation auto-compensation technique can be simply explained as follows: the rotation rate of the rotary table is denoted by $w$; if the aircraft keeps the same flight posture during one rotation period, $C_{b}^{n}$ will remain the constant. The rotation matrix $\boldsymbol{C}_{b}^{n}$ can be described as

$$
\left.\begin{array}{cc}
-\cos \varphi \sin \psi & \sin \theta \cos \psi+\cos \theta \sin \varphi \sin \psi \\
\cos \varphi \cos \psi & \sin \theta \sin \varphi-\cos \theta \sin \varphi \cos \psi \\
\sin \varphi & \cos \theta \cos \varphi
\end{array}\right] .
$$


The gyro drifts are expressed in the n-frame as

$$
\begin{gathered}
\varepsilon^{n}=\boldsymbol{C}_{b}^{n} \boldsymbol{C}_{p}^{b} \varepsilon^{p}= \\
\boldsymbol{C}_{b}^{n}\left[\begin{array}{c}
\left(\varepsilon_{x}^{p} \cos \theta_{b p}+\varepsilon_{z}^{p} \sin \theta_{b p}\right) \cos (w t)+\varepsilon_{y}^{p} \sin (w t) \\
\left(\varepsilon_{x}^{p} \cos \theta_{b p}+\varepsilon_{z}^{p} \sin \theta_{b p}\right) \sin (w t)+\varepsilon_{y}^{p} \cos (w t) \\
-\varepsilon_{x}^{p} \sin \theta_{b p}+\varepsilon_{z}^{p} \cos \theta_{b p}
\end{array}\right]
\end{gathered}
$$

where $\varepsilon^{n}=\left[\begin{array}{lll}\varepsilon_{E} & \varepsilon_{N} & \varepsilon_{U}\end{array}\right]^{\mathrm{T}}$ and $\varepsilon^{p}=\left[\begin{array}{lll}\varepsilon_{x} & \varepsilon_{y} & \varepsilon_{z}\end{array}\right]^{\mathrm{T}}$ represent the gyroscope drifts expressed in the $n$-frame and in the p-frame, respectively.

The integration of the biases in a complete cycle can be viewed as

$$
\begin{array}{r}
\int_{0}^{T^{\prime}} \varepsilon^{n} \mathrm{~d} t=\int_{0}^{T^{\prime} / 2} \boldsymbol{C}_{p}^{n} \boldsymbol{C}_{p}^{b} \varepsilon^{p} \mathrm{~d} t+\int_{T^{\prime} / 2}^{T^{\prime}} \boldsymbol{C}_{p}^{n} \boldsymbol{C}_{p}^{b} \varepsilon^{p} \mathrm{~d} t= \\
\boldsymbol{C}_{b}^{n}\left[\begin{array}{c}
0 \\
0 \\
T^{\prime}\left(-\varepsilon_{x}^{p} \sin \theta_{b p}+\varepsilon_{z}^{\rho} \cos \theta_{k p}\right)
\end{array}\right]
\end{array}
$$

where $T^{\prime}=2 \frac{2 \pi}{w}$ represents the rotation period. From (7), it can be known that the result of rotation modulation is related to the angle of $\theta_{b p}$. When the angle of $\theta_{b p}$ is $45^{\circ}$ and the drifts of three gyros are the same, the errors of gyros can be modulated into zero in theory.

\subsection{Principle of CNS}

CNS is mainly composed of the star sensor and the star image processing software. The starlight vector in the sframe $s_{s}$ can be acquired [21] after preprocessing of the original star image captured by the star sensor. Meanwhile, after star image pattern recognition, the starlight vector expressed in the earth-centered inertial frame $s_{i}$ can also be obtained [22] as

$$
\boldsymbol{s}_{i}=\left[\begin{array}{lll}
\cos \Delta \cos R_{A} & \cos \Delta \sin R_{A} & \sin \Delta
\end{array}\right]^{\mathrm{T}}
$$

where $\Delta$ and $R_{A}$ are the declination and right ascension of the star, respectively.

The star altitude is a function of the aircraft's position and the star's geographic position (GP) [23], which is expressed as

$$
\left\{\begin{array}{l}
\sin H=\sin L \sin \Delta+\cos L \cos \Delta \cos t_{L H A} \\
t_{L H A}=t_{G H A}+\lambda
\end{array}\right.
$$

where $H$ is the star altitude, $t_{L H A}$ is the local hour angle of the star, $\lambda$ plus $t_{G H A}$ makes $t_{L H A} . t_{G H A}$ is the angular distance to the GP's meridian measured westward from the $0^{\circ}$ longitude. The GP of a star can be determined by its $\Delta$ and $t_{L H A}$, which can be obtained with the astronomical almanac and a precise time.

\section{System model of the tightly coupled rotational SINS/CNS integrated navigation}

The rotational SINS/CNS integrated navigation algorithm is mainly composed of three parts: the rotational SINS, the CNS and the integrated navigation algorithm, as shown in Fig. 3. The rotational SINS can calculate the aircraft's position, velocity, and attitude with measurements from gyroscopes and accelerometers. In the rotational SINS, rotation modulation technology can modulate the biases of accelerometers and gyros drifts into a sine or cosine function [12], which can improve the horizontal reference's accuracy. The starlight vector is used as a measurement, and the star altitude can be provided with the help of the horizontal reference, which also is used as a measurement. Through the state model and measurement model of the established rotational SINS/CNS integrated navigation, the estimated position, velocity and attitude can be obtained.

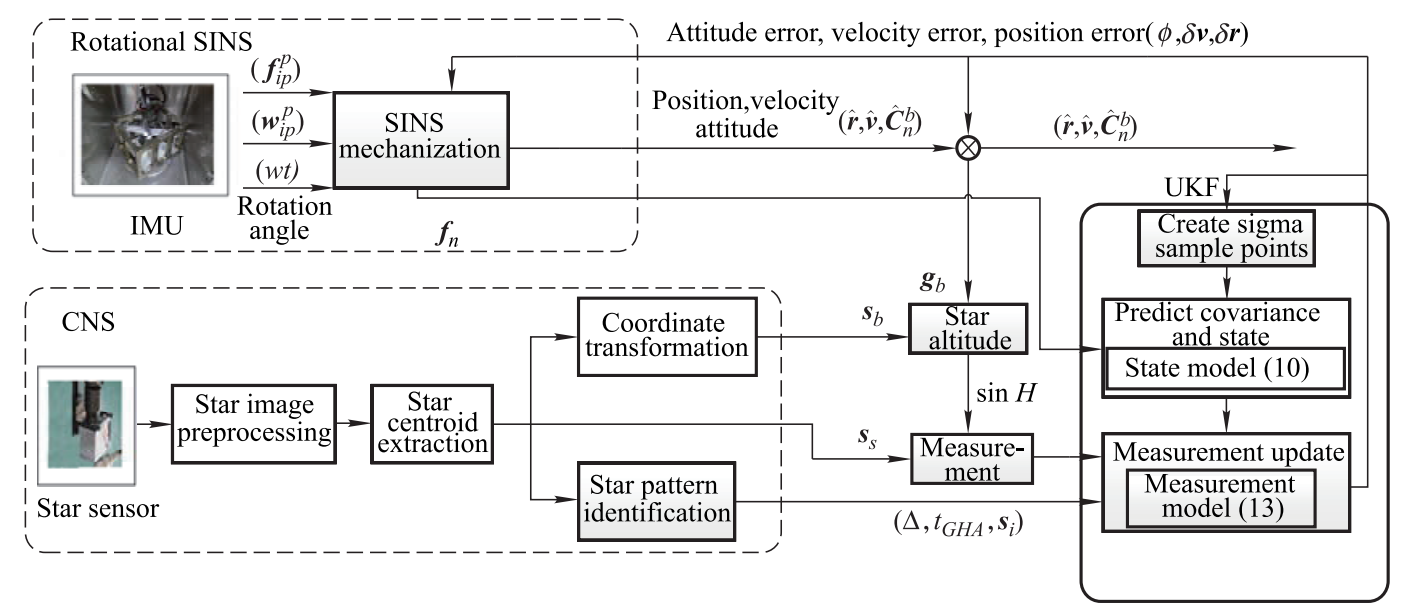

Fig. 3 Workflow of the tightly coupled rotational SINS/CNS integrated navigation algorithm 


\subsection{State model}

Because the altitude of the SINS is divergent, and the CNS cannot provide the altitude either, the altimeter is used to obtain the altitude information. The state model does not contain altitude information. The rotational SINS error equation in the navigation frame is used as a state model, which can be obtained from (1) as

$$
\begin{aligned}
& \left\{\begin{array}{l}
\dot{\boldsymbol{\phi}}=\boldsymbol{\phi} \times \boldsymbol{\omega}_{i n}^{n}+\delta \boldsymbol{\omega}_{i n}^{n}-\boldsymbol{C}_{p}^{n} \boldsymbol{\varepsilon}^{p} \\
\delta \dot{\boldsymbol{v}}_{n}=-\boldsymbol{\phi} \times \boldsymbol{f}_{n}-\left(2 \delta \boldsymbol{\omega}_{i e}^{n}+\delta \boldsymbol{\omega}_{e n}^{n}\right) \times \\
\boldsymbol{v}_{n}-\left(2 \boldsymbol{\omega}_{i e}^{n}+\boldsymbol{\omega}_{e n}^{n}\right) \times \delta \boldsymbol{v}_{n}+\boldsymbol{C}_{p}^{n} \boldsymbol{\nabla}^{p} \\
\delta \dot{\boldsymbol{r}}_{n}=\boldsymbol{R} \delta \boldsymbol{v}_{n}+\boldsymbol{D} \delta \boldsymbol{r}_{n} \\
\dot{\boldsymbol{\varepsilon}}^{p}=0 \\
\dot{\boldsymbol{\nabla}}^{p}=0
\end{array}\right. \\
& \boldsymbol{R}=\left[\begin{array}{cc}
0 & 1 / R_{y} \\
1 /\left(R_{x} \cos L\right) & 0
\end{array}\right] \\
& \boldsymbol{D}=\left[\begin{array}{cc}
\left(v_{E}^{n} / R_{x}\right) \sec L \tan L & 0 \\
0 & 0
\end{array}\right]
\end{aligned}
$$

where $\phi=\left[\begin{array}{lll}\phi_{E} & \phi_{N} & \phi_{U}\end{array}\right]^{\mathrm{T}}$ represents the misalignment angle, $\delta \boldsymbol{v}_{n}=\left[\begin{array}{ll}\delta v_{E} & \delta v_{N}\end{array}\right]^{\mathrm{T}}$ represents the velocity error, $\delta \boldsymbol{r}_{n}=\left[\begin{array}{ll}\delta L & \delta \lambda\end{array}\right]^{\mathrm{T}}$ represents the position error, $\nabla^{p}=\left[\begin{array}{ccc}\Delta_{x}^{p} & \Delta_{y}^{p} & \Delta_{z}^{p}\end{array}\right]^{\mathrm{T}}$ represents the accelerometer biases expressed in the p-frame, $\boldsymbol{f}^{n}=\boldsymbol{C}_{p}^{n} \boldsymbol{f}_{i p}^{p}$ represents the projection of the output of accelerometers in the n-frame, $\delta \boldsymbol{w}_{i e}^{n}=\left[\begin{array}{lll}0 & -w_{i e} \sin L \delta L & w_{i e} \cos L \delta L\end{array}\right]^{\mathrm{T}}$ denotes the error of $\boldsymbol{w}_{i e}$ expressed in the $\mathrm{n}$-frame, and

$$
\delta \boldsymbol{w}_{e n}^{n}=\left[-\frac{\delta v_{N}^{n}}{R_{y}} \frac{\delta v_{E}^{n}}{R_{x}} \frac{\delta v_{E}^{n}}{R_{x}} \tan L+\frac{v_{E}^{n}}{R_{x}} \sec ^{2} L\right]^{\mathrm{T}}
$$

denotes the error of $\boldsymbol{w}_{e n}$ expressed in the $\mathrm{n}$-frame.

\subsection{Measurement model}

The starlight vector and star altitude are used as the measurement in this tightly coupled SINS/CNS integrated navigation system.

\subsubsection{Measurement model of starlight vector}

From the CNS's principle, the starlight vector can be obtained in the i-frame and s-frame. The measurement equation can be expressed as

$$
\begin{gathered}
\boldsymbol{s}_{s}=\boldsymbol{C}_{b}^{s} \boldsymbol{C}_{n}^{b} \boldsymbol{C}_{n}^{n} \boldsymbol{C}_{e}^{n} \boldsymbol{C}_{i}^{e} \boldsymbol{s}_{i} \\
\boldsymbol{C}_{i}^{e}=\left(\boldsymbol{C}_{e}^{i}\right)^{\mathrm{T}}=\left[\begin{array}{ccc}
\cos t_{S H A} & \sin t_{S H A} & 0 \\
-\sin t_{S H A} & \cos t_{S H A} & 0 \\
0 & 0 & 1
\end{array}\right] \\
\boldsymbol{C}_{e}^{n}=\left(\boldsymbol{C}_{n}^{e}\right)^{\mathrm{T}}=\left[\begin{array}{ccc}
-\sin \lambda & \cos \lambda & 0 \\
-\sin L \cos \lambda & -\sin L \sin \lambda & \cos L \\
\cos L \cos \lambda & \cos L \sin \lambda & \sin L
\end{array}\right]
\end{gathered}
$$

where $C_{b}^{s}$ is the installment matrix of the star sensor, $C_{i}^{e}$ is the transformation matrix from the i-frame to the eframe, and $t_{S H A}$ is the Greenwich hour angle of the vernal equinox, which can be obtained from the astronomical almanac with precise time measurement, $C_{c}^{n}$ is the transformation matrix from the e-frame to the n-frame, $C_{n}^{b}$ is the attitude matrix provided by the SINS, $C_{n}^{n^{\prime}}$ is the attitude error matrix, which can be written [1] as

$$
\boldsymbol{C}_{n}^{n}=[\boldsymbol{I}-\boldsymbol{\phi}]=\left[\begin{array}{ccc}
1 & -\phi_{z} & \phi_{y} \\
\phi_{z} & 1 & \phi_{x} \\
-\phi_{y} & -\phi_{x} & 1
\end{array}\right]
$$

\subsubsection{Measurement model of star altitude}

The star altitude can be obtained using the horizon reference provided by the accelerometers and the starlight vector provided by the star sensor. Using $\sin H$ as the measurement, from (9), the star altitude's measurement equation can be written as

$$
\begin{gathered}
\sin H=\sin (L+\delta L) \sin \Delta+ \\
\cos (L+\delta L) \cos \Delta \cos \left(t_{G H A}+\lambda+\delta \lambda\right) .
\end{gathered}
$$

According to (11) and (13), the measurement using $Z=$ $\left[\begin{array}{ll}\boldsymbol{s}_{s} & \sin H\end{array}\right]^{\mathrm{T}}$ can be expressed as

$$
\boldsymbol{Z}=h(\boldsymbol{X})+\boldsymbol{V}
$$

where $\boldsymbol{V}$ is the measurement noise. Because the measurement model (11) is nonlinear, the unscented Kalman filter (UKF) [24,25] is implemented to fuse rotational SINS and CNS data in this study.

\section{Simulations and analysis}

In this section, semi-physical simulation experiments are performed to test the performance of this tightly coupled rotational SINS/CNS integrated navigation method.

\subsection{Semi-physical simulation system}

All the simulation data is generated through this semiphysical simulation system shown in Fig. 4. This system is composed of a path generator, a rotational SINS, a CNS simulation system and the SINS/CNS integrated navigation system. The path generator is used to produce the ideal path of the aircraft, including the true position, the velocity, the attitude and the IMU output. The rotational SINS contains an IMU and a rotator assembly. We keep the aircraft stationary to collect IMU's noises. Then, the IMU's noises are added to the ideal values to get the simulated IMU data. The CNS simulation system is composed of a star light simulator and a star sensor. The star light simulator produces the simulated star light, which contains two 
parts: a star map simulation PC and a collimator. According to the optical axis direction of the star sensor and the aircraft's position and attitude, the star map simulation PC can generate the simulated star map [26]. A collimator is a photoelectric conversion device, which can convert the star map into infinitely parallel starlight, which can then be captured by the star sensor [27]. Then, the starlight vectors in the Earth's inertial frame and in the s-frame can be obtained by star image preprocessing, star centroid extraction, and pattern recognition.

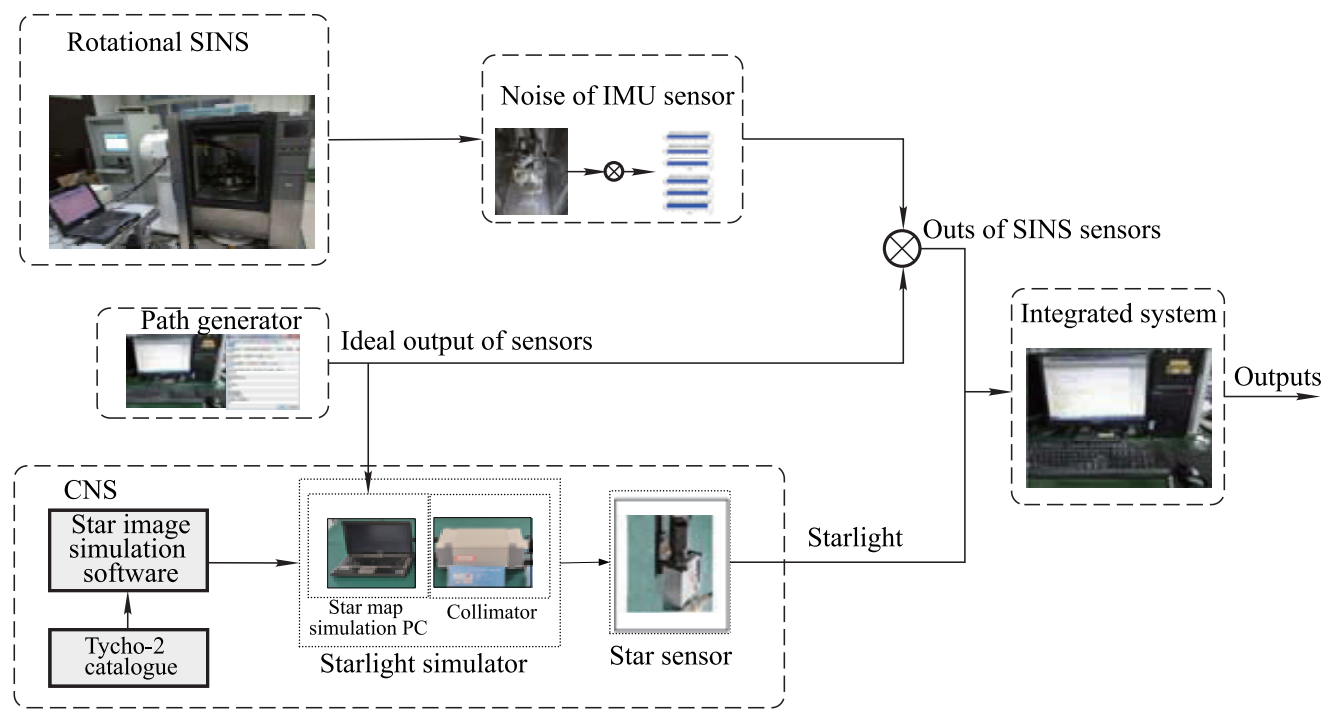

Fig. 4 Semi-physical simulation system

\subsection{Simulation condition}

The flight path used in the following simulation is predicted in Fig.5. The total running time is $4 \mathrm{~h}$ and the initial position of the aircraft is longitude $116^{\circ} \mathrm{E}$, latitude $40{ }^{\circ} \mathrm{N}$, and altitude $10 \mathrm{~km}$. Eastern velocity is $150 \mathrm{~m} / \mathrm{s}$. The yaw angle, pitch angle and roll angle are $270^{\circ}, 0^{\circ}$, and $0^{\circ}$ respectively. The initial errors in longitude and latitude are both $10 \mathrm{~m}$, and the altitude channel is damped by the altimeter. The initial velocity errors of eastern direction and northern direction are both $0.2 \mathrm{~m} / \mathrm{s}$. The initial misalignment angles are $10^{\prime \prime}$ in eastern and northern directions and $20^{\prime \prime}$ in the zenithal direction.

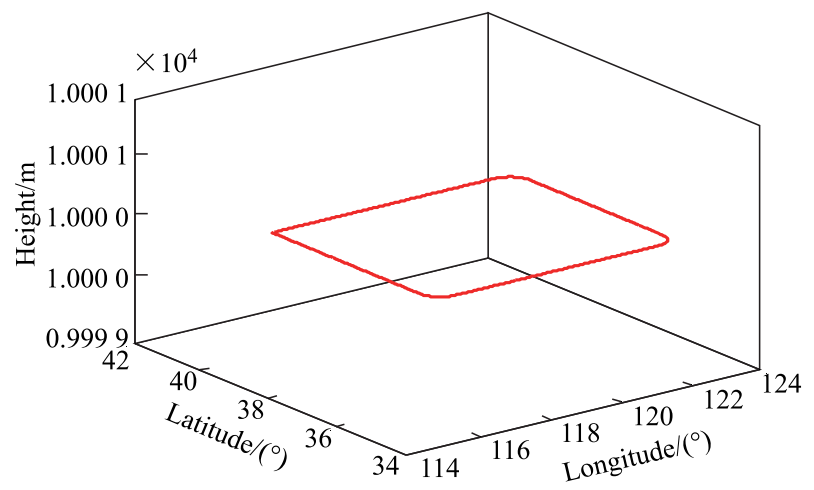

(a) Path
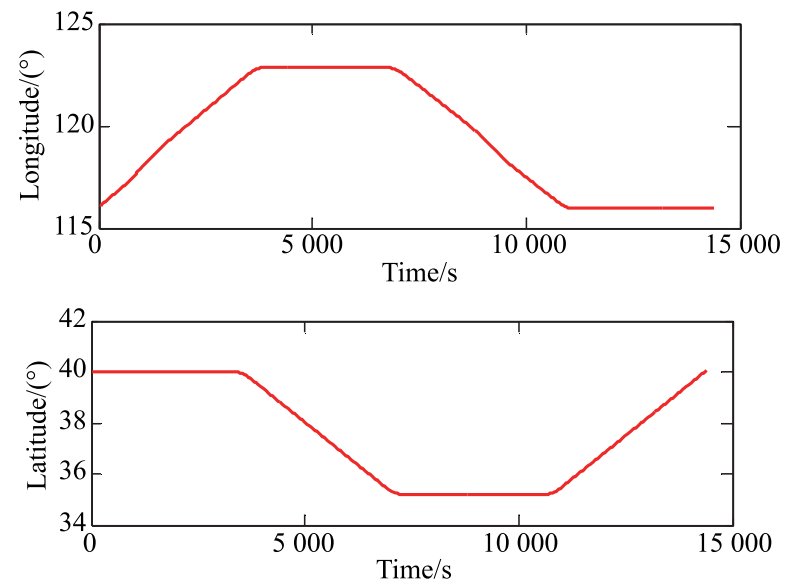

(b) Position
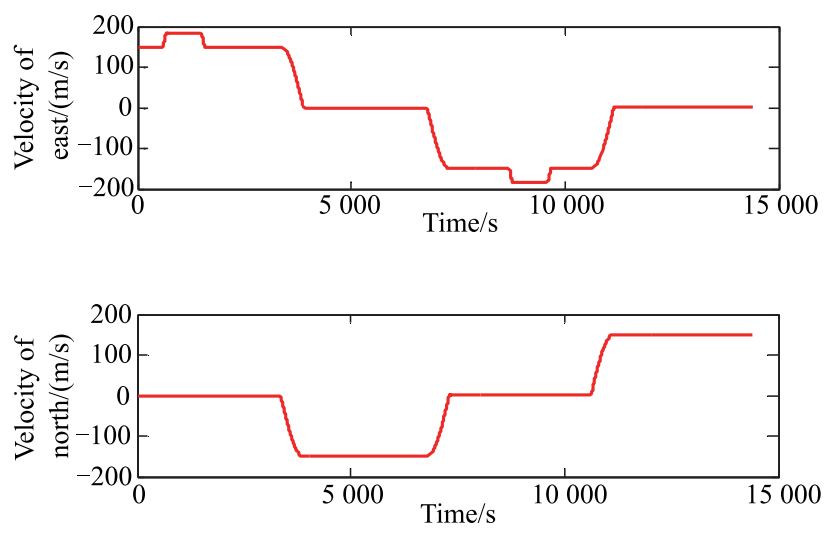

(c) Velocity 

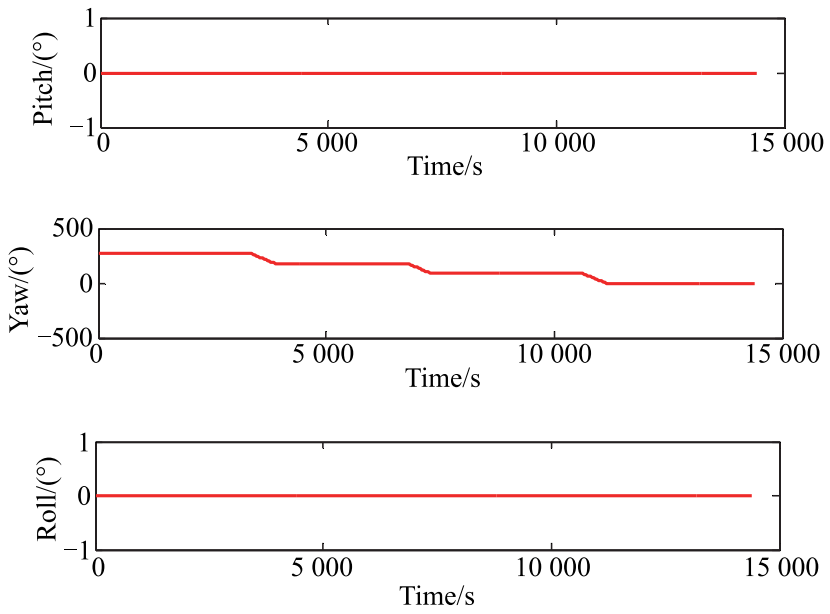

(d) Attitude

Fig. 5 Ideal path of the aircraft

The drift of each gyro is $0.005^{\circ} / \mathrm{h}$. The bias of each accelerometer is $50 \mu \mathrm{g}$, with a frequency of $100 \mathrm{~Hz}$. The error characteristics of the accelerometers and gyroscopes are shown in Fig. 6. As can be seen in these pictures, measurement errors of all sensors contain two types: system errors and random errors. The star sensor's accuracy is $3^{\prime \prime}(1 \sigma)$ with a frequency of $5 \mathrm{~Hz}$. The observed star is changed every $10 \mathrm{~min}$. The filtering period is $1 \mathrm{~s}$. The IMU keeps rolling around the $z_{b}$ axis. The constant angular rate $w$ is $6 \%$. The constant $\theta_{b p}$ is zero.
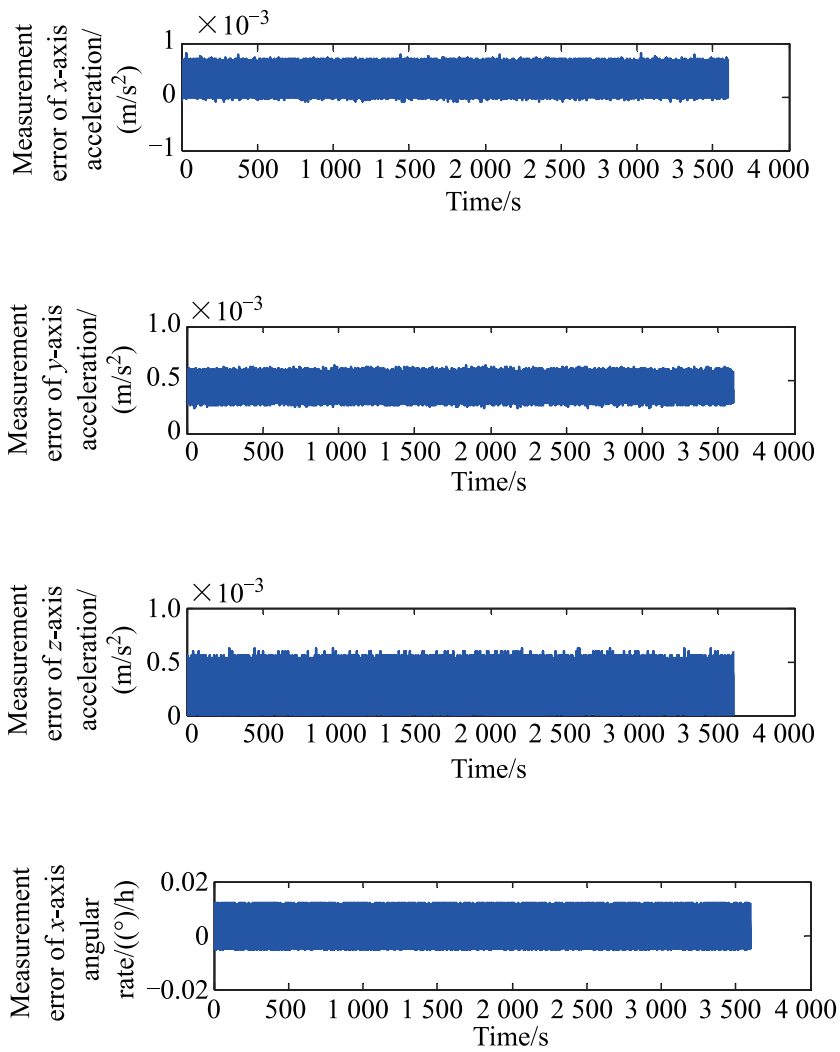
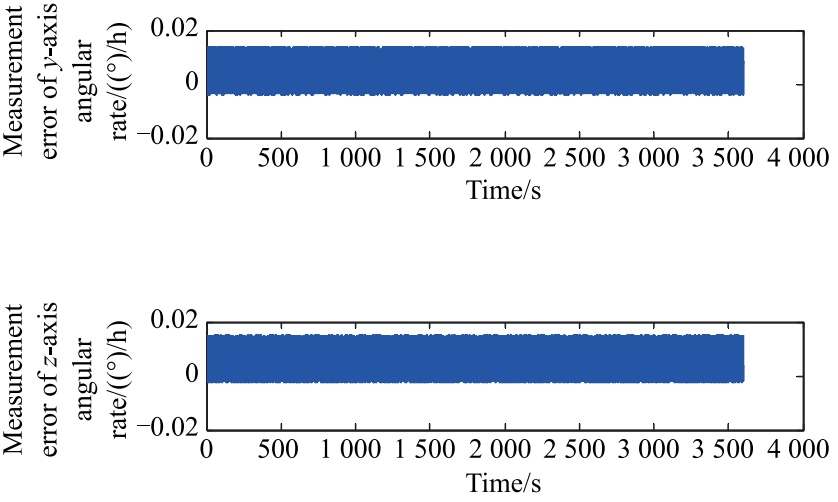

Fig. 6 Errors of the accelerometers and gyros

\subsection{Comparison of simulation results}

The navigation simulation results are shown in this section, including the SINS navigation, the rotational SINS navigation, the tightly coupled SINS/CNS integrated navigation and the tightly coupled rotational SINS/CNS integrated navigation.

\subsubsection{SINS}

The navigation results of the SINS under the above simulation conditions are shown in Fig. 7 and Table 1. From these results, we can see that the estimation errors of position, velocity, and attitude increase rapidly over time, which is caused by the continuous integration of gyros bias and accelerometers bias. During the simulation period, the mean estimation errors in pitch, roll, and yaw are 14.43", 18.66", and $59.20^{\prime \prime}$, respectively. The mean estimation eastern velocity errors and north velocity errors are $0.66 \mathrm{~m} / \mathrm{s}$ and $0.60 \mathrm{~m} / \mathrm{s}$, respectively. The mean longitude estimation error and latitude estimation error are $924.39 \mathrm{~m}$ and 820.53 $\mathrm{m}$, respectively.
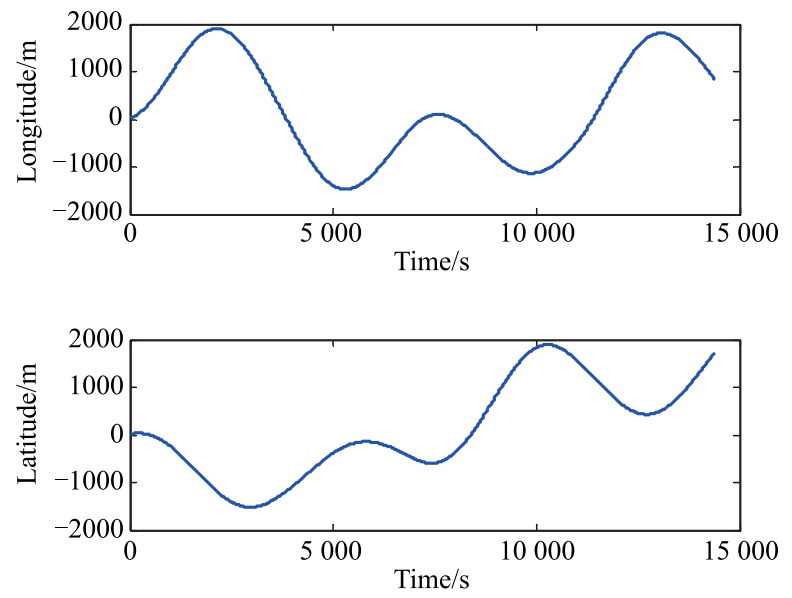

(a) Position error 

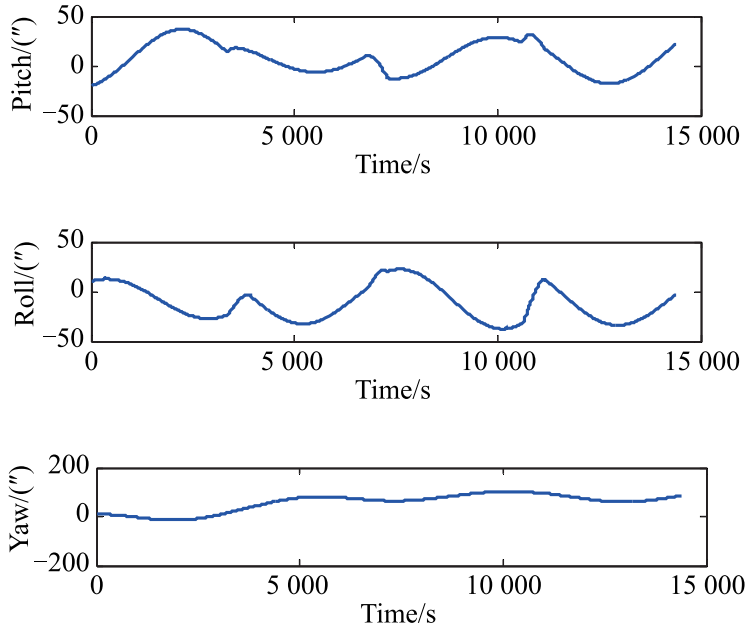

(b) Attitude error
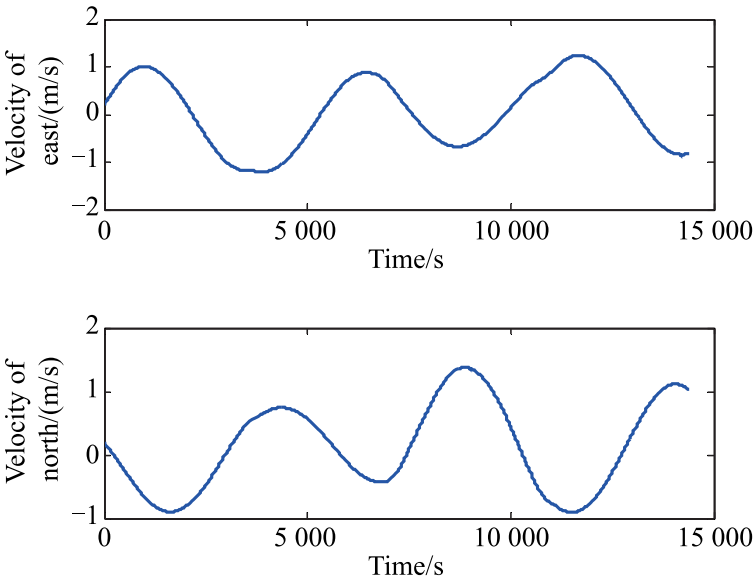

(c) Velocity error

Fig. 7 Simulation results of the SINS

Table 1 Results of the different navigation methods

\begin{tabular}{|c|c|c|c|c|c|c|c|c|c|}
\hline \multirow{2}{*}{ Method } & \multicolumn{2}{|c|}{ Mean position error/m } & \multicolumn{2}{|c|}{ Root mean square/m } & \multicolumn{3}{|c|}{ Mean attitude error $/\left({ }^{\prime \prime}\right)$} & \multicolumn{2}{|c|}{ Mean velocity error $/(\mathrm{m} / \mathrm{s})$} \\
\hline & Longitude & Latitude & Longitude & Latitude & Pitch & Roll & Yaw & Eastern & Northern \\
\hline SINS & 924.39 & 820.53 & 547.85 & 526.16 & 14.43 & 18.66 & 59.20 & 0.66 & 0.60 \\
\hline Rotational SINS & 786.85 & 313.39 & 505.08 & 218.87 & 10.05 & 10.10 & 43.38 & 0.43 & 0.39 \\
\hline $\begin{array}{l}\text { The tightly coupled SINS/CNS } \\
\text { integrated navigation method }\end{array}$ & 234.21 & 173.55 & 113.16 & 100.32 & 4.98 & 6.01 & 5.46 & 0.18 & 0.16 \\
\hline $\begin{array}{l}\text { The tightly coupled rotational } \\
\text { SINS/CNS integrated navigation }\end{array}$ & 33.55 & 33.52 & 27.11 & 22.02 & 1.24 & 0.96 & 0.97 & 0.04 & 0.06 \\
\hline
\end{tabular}

\subsubsection{Rotational SINS}

The navigation results of the rotational SINS are shown in Fig. 8 and Table 1. As shown in Fig. 8, the estimation errors, including position error, velocity error, and attitude error, still increase rapidly over time, but less than that of the SINS navigation results. This is because the rotation modulation technology cannot modulate the initial system errors [28]. The mean estimation error in longitude and latitude are $786.85 \mathrm{~m}$ and $313.39 \mathrm{~m}$, respectively.
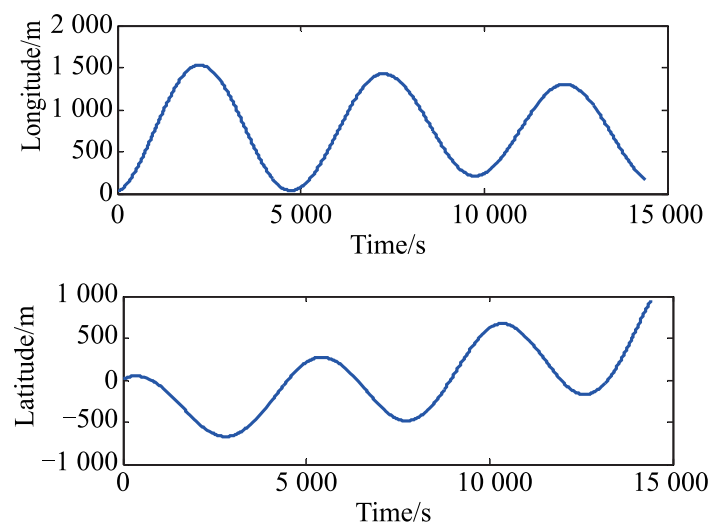

(a) Position error

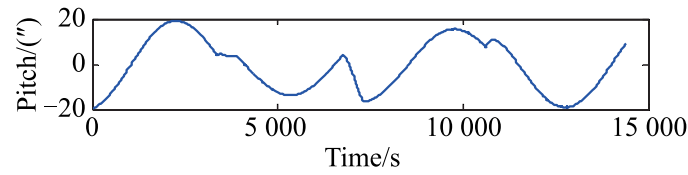

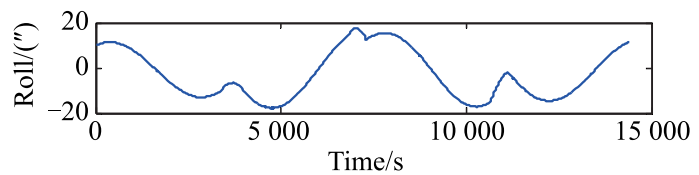

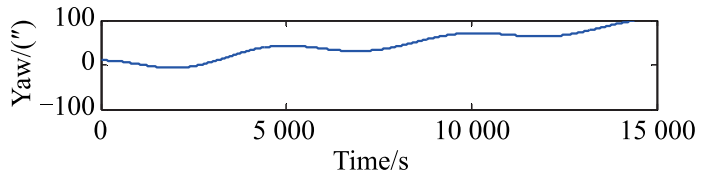

(b) Attitude error
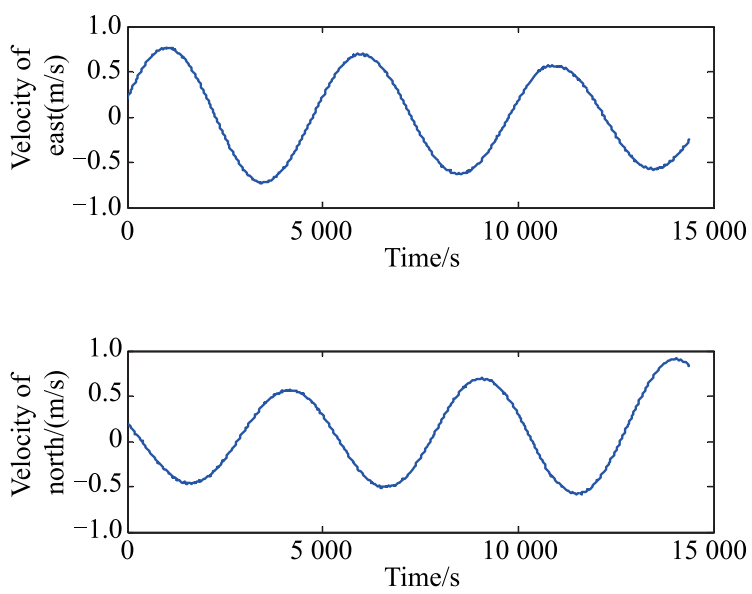

(c) Velocity error

Fig. 8 Simulation results of rotational SINS 


\subsubsection{The tightly coupled SINS/CNS integrated navigation method}

The navigation results of the tightly coupled SINS/CNS integrated navigation are shown in Fig. 9 and Table 1. As shown in Fig. 9, the estimation errors which include position error, velocity error and attitude error are much better than that of the SINS and rotational SINS navigation method. However, the error of horizon reference provided by the SINS still grows with time, which will introduce error in star altitude and affect the estimation of position. Because the attitude matrix is easily affected by the position matrix [7], the estimation errors of attitude are also cumulated with the time. The mean estimation error in longitude and latitude of the tightly coupled SINS/CNS integrated navigation are $234.21 \mathrm{~m}$ and $173.55 \mathrm{~m}$, which are $25.34 \%$ and $21.15 \%$ of the SINS, respectively.
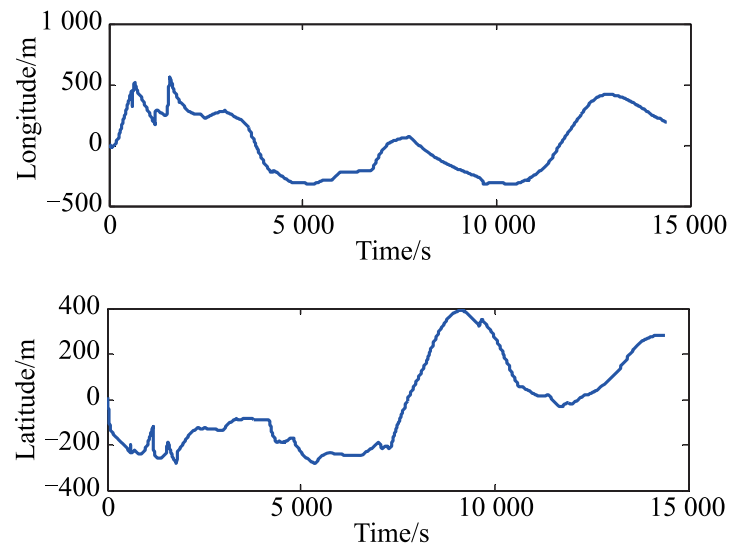

(a) Position error
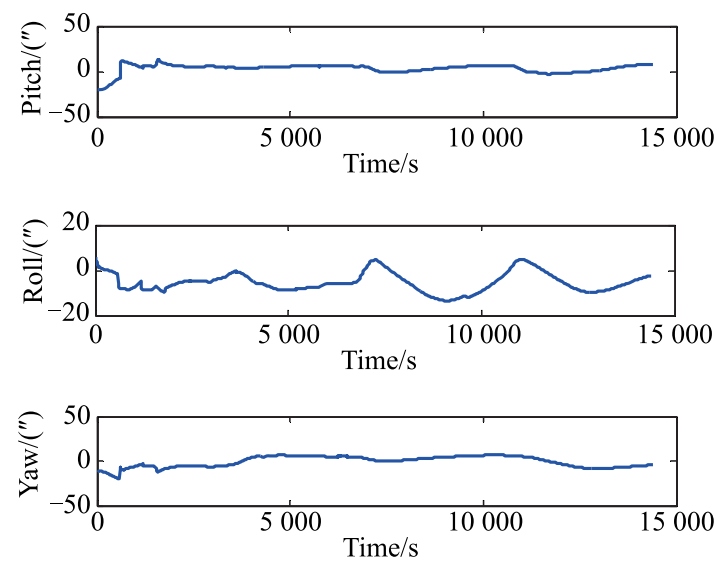

(b) Attitude error

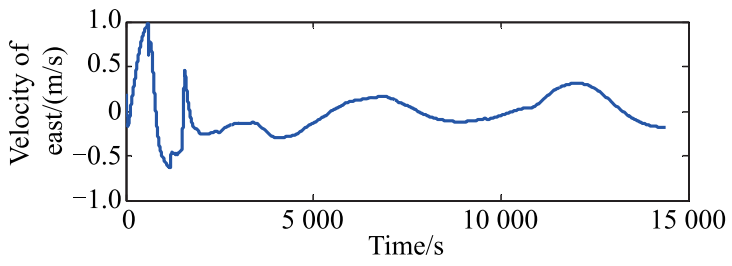

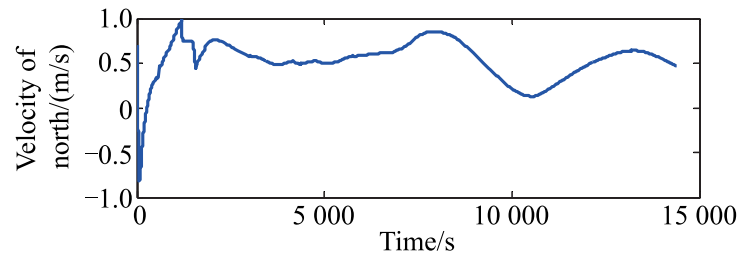

(c) Velocity error

Fig. 9 Simulation results of the tightly coupled SINS/CNS integrated navigation

\subsubsection{The tightly coupled rotational SINS/CNS integrated navigation}

The navigation results of the tightly coupled rotational SINS/CNS integrated navigation are shown in Fig. 10 and Table 1. As shown in Fig. 10, the position, attitude, and velocity can all be estimated correctly with the assistant of the starlight vector and star altitude, and the attitude estimation curves converge quickly. Using the rotation modulation techniques, horizon reference provided by the rotational SINS is more precise, which leads to a decrease of the position error of the tightly coupled rotational SINS/CNS integrated navigation. The mean estimation errors in pitch, roll, and yaw are $1.24^{\prime \prime}, 0.96^{\prime \prime}$ and $0.97^{\prime \prime}$, respectively. The mean eastern estimation velocity error and northern estimation velocity error are $0.04 \mathrm{~m} / \mathrm{s}$ and $0.06 \mathrm{~m} / \mathrm{s}$, respectively. The mean estimation errors in longitude and latitude are $33.55 \mathrm{~m}$ and $33.52 \mathrm{~m}$, respectively. Compared with above navigation methods, the mean estimation errors in longitude and latitude of the tightly coupled rotational SINS/CNS integrated navigation are about $4.26 \%$ and $10.70 \%$ of the rotational SINS, $14.32 \%$ and $19.31 \%$ of the tightly coupled SINS/CNS integrated navigation, respectively. From these results, we can see that it is better to use the tightly coupled rotational SINS/CNS integrated navigation for aircraft. This method can not only correct the attitude errors, but also restrain the errors of position and velocity in a limited range.
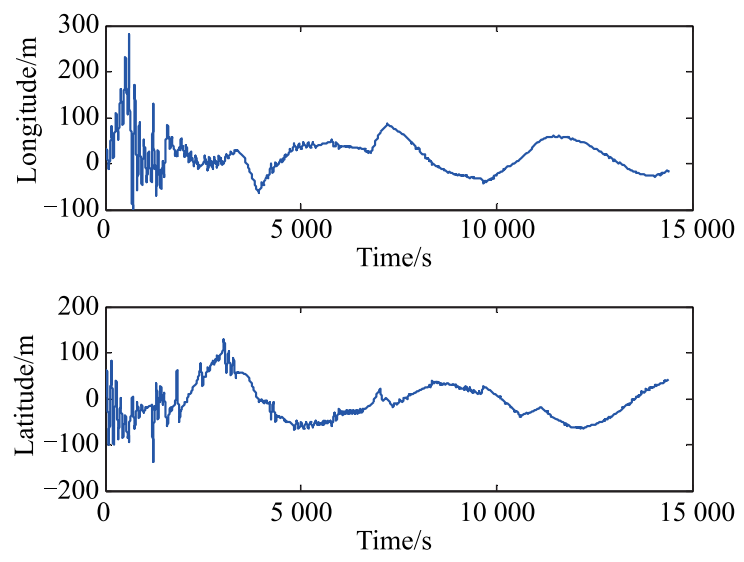

(a) Position error 

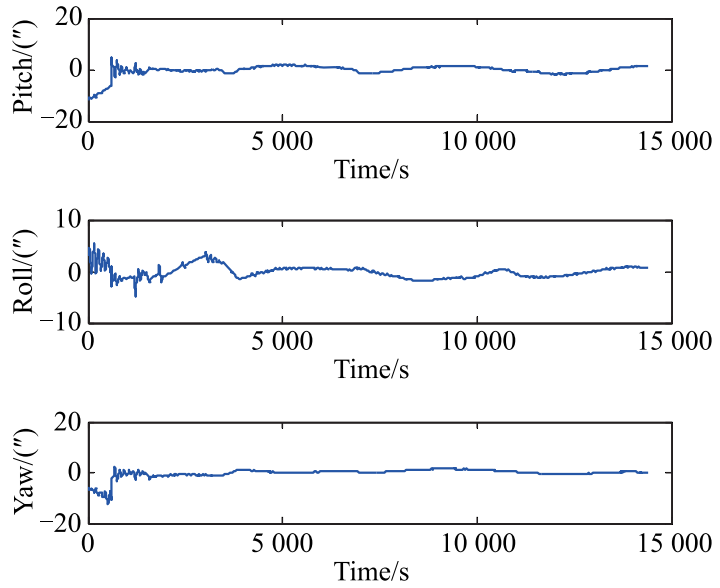

(b) Attitude error
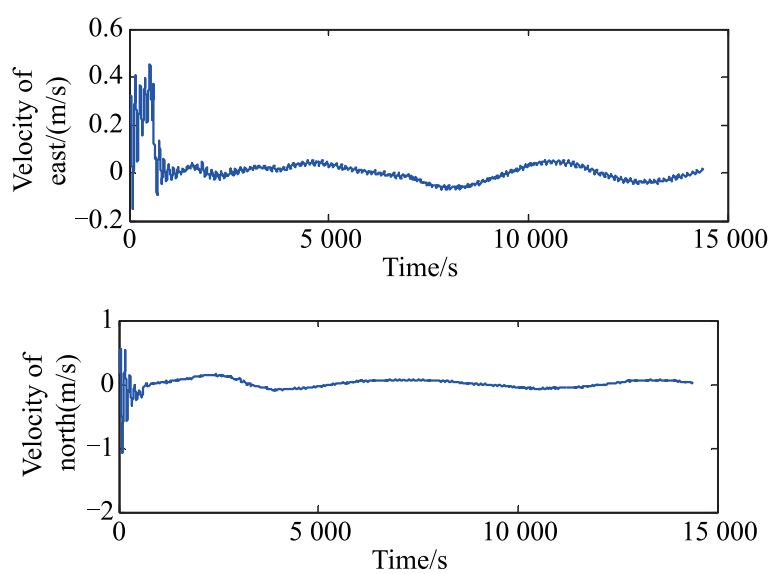

(c) Velocity error

Fig. 10 Simulation results of the tightly coupled rotational SINS/CNS integrated navigation

\subsection{Impact factors}

This part analyzes the impacts of the rotation scheme, the gyros' accuracy, the accelerometers' accuracy, star sensor's accuracy, the IMU installation errors, and the star sensor installation errors on the navigation performance of the tightly coupled rotational SINS/CNS integrated navigation.

(i) The impact of the installation angle $\theta_{b p}$

To analyze the impact of installation angle $\theta_{b p}$ on the tightly coupled rotational SINS/CNS integrated navigation, the initial attitude error, the velocity error, and the position error of the integrated system are set as zero to avoid their influence. The detailed results are shown in Table 2. They show that when $\theta_{b p}$ is $45^{\circ}$, the tightly coupled rotational SINS/CNS integrated navigation achieves the highest accuracy. However, initial errors are inevitable in reality, so we set the same simulation conditions as in Section 4.4. The detailed results under this condition are shown in Table 3 . The navigation accuracies of this method with different installation angles are almost the same. Since the rotation modulation cannot modulate initial errors, the best performance cannot be obtained when $\theta_{b p}$ is $45^{\circ}$ if there are initial attitude error, velocity error and position error of the integrated system. In addition, if the drifts of each gyro and accelerometer in three directions are not the same, the best performance cannot be obtained when $\theta_{b p}$ is $45^{\circ}$, either. To sum up, the installation angle of the IMU has little influence on the integrated navigation.

Table 2 Results of the integrated navigation with different $\theta_{b p}$ without initial errors

\begin{tabular}{|c|c|c|c|c|c|c|c|}
\hline \multirow{2}{*}{$\theta_{b p} /\left(^{\circ}\right)$} & \multicolumn{2}{|c|}{ Mean position error $/ \mathrm{m}$} & \multicolumn{3}{|c|}{ Mean attitude error $/\left({ }^{\prime \prime}\right)$} & \multicolumn{2}{|c|}{ Mean velocity error/(m/s) } \\
\hline & Longitude & Latitude & Pitch & Roll & Yaw & Eastern & Northern \\
\hline 0 & 20.51 & 11.94 & 0.16 & 0.24 & 0.60 & 0.01 & 0.02 \\
\hline 30 & 17.05 & 10.46 & 0.11 & 0.17 & 0.53 & 0.01 & 0.01 \\
\hline 45 & 13.24 & 6.94 & 0.11 & 0.14 & 0.49 & 0.01 & 0.01 \\
\hline 60 & 15.37 & 8.28 & 0.13 & 0.14 & 0.48 & 0.01 & 0.01 \\
\hline 90 & 18.52 & 10.46 & 0.15 & 0.16 & 0.46 & 0.01 & 0.01 \\
\hline
\end{tabular}

Table 3 Results of the integrated navigation with different $\theta_{b p}$ with initial errors

\begin{tabular}{|c|c|c|c|c|c|c|c|}
\hline \multirow{2}{*}{$\theta_{b p} /\left(^{\circ}\right)$} & \multicolumn{2}{|c|}{ Mean position error $/ \mathrm{m}$} & \multicolumn{3}{|c|}{ Mean attitude error $/\left({ }^{\prime \prime}\right)$} & \multicolumn{2}{|c|}{ Mean velocity error $/(\mathrm{m} / \mathrm{s})$} \\
\hline & Longitude & Latitude & Pitch & Roll & Yaw & Eastern & Northern \\
\hline 0 & 33.55 & 33.52 & 1.24 & 0.96 & 0.97 & 0.04 & 0.06 \\
\hline 30 & 34.75 & 34.64 & 1.27 & 0.98 & 0.94 & 0.04 & 0.06 \\
\hline 45 & 34.96 & 35.16 & 1.26 & 0.10 & 0.90 & 0.04 & 0.06 \\
\hline 60 & 35.67 & 35.92 & 1.28 & 1.01 & 0.94 & 0.04 & 0.06 \\
\hline 90 & 36.83 & 37.54 & 1.31 & 1.05 & 0.94 & 0.04 & 0.06 \\
\hline
\end{tabular}

(ii) The impact of gyro's accuracy

In order to illustrate the impact of the gyro drifts on the tightly coupled rotational SINS/CNS integrated navigation, the gyro drifts are set from $0.0005^{\circ} / \mathrm{h}$ to $0.01^{\circ} / \mathrm{h}$, and the other simulation conditions are the same as Section 4.4.
The detailed results are shown in Table 4. The accuracy of the gyro has little effect on the tightly coupled rotational SINS/CNS integrated navigation, mainly because the gyro drifts can be automatically compensated by using the rotation modulation [12]. 
Table 4 Results of the integrated navigation with different gyro drifts

\begin{tabular}{|c|c|c|c|c|c|c|c|}
\hline \multirow{2}{*}{$\begin{array}{c}\text { Gyro } \\
\text { accuracy } /\left(\left(^{\circ}\right) / \mathrm{h}\right)\end{array}$} & \multicolumn{2}{|c|}{ Mean position error/m } & \multicolumn{3}{|c|}{ Mean attitude error $/\left({ }^{\prime \prime}\right)$} & \multicolumn{2}{|c|}{ Mean velocity error/(m/s) } \\
\hline & Longitude & Latitude & Pitch & Roll & Yaw & Eastern & Northern \\
\hline 0.0005 & 33.49 & 33.72 & 1.22 & 0.96 & 0.96 & 0.04 & 0.06 \\
\hline 0.0025 & 33.57 & 33.44 & 1.24 & 0.96 & 0.97 & 0.04 & 0.06 \\
\hline 0.005 & 33.55 & 33.52 & 1.24 & 0.96 & 0.97 & 0.04 & 0.06 \\
\hline 0.0075 & 33.59 & 33.56 & 1.24 & 0.96 & 0.95 & 0.04 & 0.06 \\
\hline 0.01 & 33.59 & 32.62 & 1.24 & 0.96 & 0.93 & 0.04 & 0.06 \\
\hline
\end{tabular}

(iii) The impact of accelerometer's accuracy

The impact of the accelerometer biases on the tightly coupled rotational SINS/CNS integrated navigation is analyzed in this section. The biases of the accelerometer are set from $5 \mu \mathrm{g}$ to $100 \mu \mathrm{g}$. The detailed mean estimation errors are shown in Table 5. According to the data, the mean estimation errors including position error, velocity error, and attitude error, decrease slowly with the improvement of the accelerometer's accuracy. The reason is that the horizon reference is provided by the accelerometer, but the rotation modulation can only modulate the accelerometer's biases into a sine or cosine function rather than zero [12].

Table 5 Results of the integrated navigation with different accelerometer biases

\begin{tabular}{|c|c|c|c|c|c|c|c|}
\hline \multirow{2}{*}{ 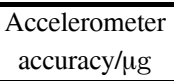 } & \multicolumn{2}{|c|}{ Mean position error/m } & \multicolumn{3}{|c|}{ Mean attitude error $/\left({ }^{\prime \prime}\right)$} & \multicolumn{2}{|c|}{ Mean velocity error $/(\mathrm{m} / \mathrm{s})$} \\
\hline & Longitude & Latitude & Pitch & Roll & Yaw & Eastern & Northern \\
\hline 5 & 32.27 & 32.18 & 1.23 & 0.92 & 0.93 & 0.03 & 0.05 \\
\hline 25 & 32.57 & 32.61 & 1.23 & 0.94 & 0.94 & 0.03 & 0.06 \\
\hline 50 & 33.55 & 33.52 & 1.24 & 0.96 & 0.97 & 0.04 & 0.06 \\
\hline 75 & 34.87 & 34.71 & 1.26 & 1.00 & 0.99 & 0.04 & 0.06 \\
\hline 100 & 36.39 & 36.15 & 1.30 & 1.05 & 1.02 & 0.04 & 0.07 \\
\hline
\end{tabular}

(iv) The impact of star sensor's accuracy

To illustrate the impact of the star sensor accuracy on the tightly coupled rotational SINS/CNS integrated navigation, the accuracy of the star sensor is set from $2^{\prime \prime}$ to $6^{\prime \prime}$. The detailed results are shown in Table 6. The data from the table shows the star sensor's accuracy has impacts on the integrated navigation. The higher the star sensor's accuracy, the smaller the mean estimation errors of the tightly coupled rotational SINS/CNS integrated navigation. This is because the accuracy of the CNS strongly depends on the star sensor's accuracy [2].

Table 6 Results of the integrated navigation with different star sensor accuracies

\begin{tabular}{|c|c|c|c|c|c|c|c|}
\hline \multirow{2}{*}{$\begin{array}{c}\text { Star sensor } \\
\text { accuracy/("') }\end{array}$} & \multicolumn{2}{|c|}{ Mean position error/m } & \multicolumn{3}{|c|}{ Mean attitude error $/\left({ }^{\prime \prime}\right)$} & \multicolumn{2}{|c|}{ Mean velocity error $/(\mathrm{m} / \mathrm{s})$} \\
\hline & Longitude & Latitude & Pitch & Roll & Yaw & Eastern & Northern \\
\hline 2 & 31.48 & 33.11 & 1.22 & 0.96 & 0.87 & 0.04 & 0.06 \\
\hline 3 & 33.55 & 33.52 & 1.24 & 0.96 & 0.97 & 0.04 & 0.06 \\
\hline 4 & 36.44 & 34.09 & 1.26 & 0.96 & 1.09 & 0.04 & 0.06 \\
\hline 5 & 39.90 & 34.81 & 1.28 & 0.97 & 1.24 & 0.04 & 0.06 \\
\hline 6 & 43.74 & 35.87 & 1.31 & 0.98 & 1.39 & 0.04 & 0.06 \\
\hline
\end{tabular}

(v) The impact of the IMU installation errors

The IMU, which includes three orthogonal accelerometers and three orthogonal gyroscopes is mounted on the rotator assembly. Installation errors of the IMU are inevitable. To simplify the analysis, the installation errors in the three directions are set as the same. To illustrate the impact of the IMU installation errors on the tightly coupled rotational SINS/CNS integrated navigation, the installation errors are set from $0^{\prime \prime}$ to $20^{\prime \prime}$. The detailed results shown in Table 7 illustrate that as the installation errors increase, the mean estimation errors of velocity and position increase. The error of the yaw angle increases slowly and remains within a small range. The reason is that the installation errors only can be modulated to some extent through the rotation modulation, especially in the vertical direction.

(vi) The impact of the star sensor installation errors

The star sensor is a highly precise, highly reliable attitude measuring device, which is widely used in spacecraft today. When the star sensor is mounted on the aircraft body, installation errors are inevitable, which causes a misaligned star sensor vector relative to the body's $x, y$, and $z$ axes. A misaligned star sensor can cause errors in the star sensor's output, which can lead to large navigation errors. To simplify the analysis, the misalignment angle in the three directions are set as the same. To illustrate the impact of star sensor installation errors on the tightly coupled 
rotational SINS/CNS integrated navigation, the misalignment angles are set from $0^{\prime \prime}$ to $20^{\prime \prime}$. The detailed results in Table 8 show that as the misalignment angle increases, the mean estimation errors of velocity, attitude, and position increase quickly. The reason is that CNS's accuracy strongly depends on measurements' accuracy.

Table 7 Results of the integrated navigation with different IMU installation errors

\begin{tabular}{|c|c|c|c|c|c|c|c|}
\hline \multirow{2}{*}{$\begin{array}{c}\text { IMU installation } \\
\text { error/ }\left({ }^{\prime \prime}\right)\end{array}$} & \multicolumn{2}{|c|}{ Mean position error/m } & \multicolumn{3}{|c|}{ Mean attitude error $/\left({ }^{\prime \prime}\right)$} & \multicolumn{2}{|c|}{ Mean velocity error $/(\mathrm{m} / \mathrm{s})$} \\
\hline & Longitude & Latitude & Pitch & Roll & Yaw & Eastern & Northern \\
\hline 0 & 33.55 & 33.52 & 1.24 & 0.96 & 0.97 & 0.04 & 0.06 \\
\hline 5 & 41.72 & 48.61 & 4.86 & 4.59 & 1.39 & 0.05 & 0.07 \\
\hline 10 & 59.35 & 71.42 & 9.27 & 9.05 & 1.88 & 0.06 & 0.09 \\
\hline 15 & 80.89 & 95.62 & 13.75 & 13.54 & 2.40 & 0.08 & 0.11 \\
\hline 20 & 103.16 & 120.29 & 18.26 & 18.04 & 2.93 & 0.10 & 0.13 \\
\hline
\end{tabular}

Table 8 Results of the integrated navigation with different star sensor installation errors

\begin{tabular}{|c|c|c|c|c|c|c|c|}
\hline \multirow{2}{*}{$\begin{array}{l}\text { Star sensor } \\
\text { installation error/ }\left({ }^{\prime \prime}\right)\end{array}$} & \multicolumn{2}{|c|}{ Mean position error/m } & \multicolumn{3}{|c|}{ Mean attitude error $/\left({ }^{\prime \prime}\right)$} & \multicolumn{2}{|c|}{ Mean velocity error $/(\mathrm{m} / \mathrm{s})$} \\
\hline & Longitude & Latitude & Pitch & Roll & Yaw & Eastern & Northern \\
\hline 0 & 33.55 & 33.52 & 1.24 & 0.96 & 0.97 & 0.04 & 0.06 \\
\hline 5 & 126.92 & 102.56 & 1.84 & 1.41 & 6.85 & 0.06 & 0.10 \\
\hline 10 & 269.05 & 218.20 & 3.37 & 2.70 & 13.24 & 0.10 & 0.19 \\
\hline 15 & 412.17 & 335.68 & 4.96 & 4.08 & 19.74 & 0.15 & 0.29 \\
\hline 20 & 555.69 & 453.22 & 6.56 & 5.49 & 26.31 & 0.20 & 0.38 \\
\hline
\end{tabular}

\section{Conclusions}

In this paper, a tightly coupled rotational SINS/CNS integrated navigation is studied. When the IMU rotates around the axis, the precision of the horizon reference provided by the rotational SINS is improved, which greatly improves the accuracy of the tightly coupled rotational SINS/CNS integrated navigation. This method is especially suitable for aircraft working in a long time and highly accurate autonomous navigation conditions. In addition, according to the analysis of impact factors, installation errors of the IMU and the star sensor have a significant impact on the accuracy of the integrated navigation. How to reduce and compensate the installation errors is a problem that needs future study. Furthermore, this paper only analyzes the rotation scheme of a single-axis reciprocating rotation. More challenging rotation schemes, such as a dual-axis alternate rotation and dual-axis continuous rotation should be considered in future study.

\section{Acknowledgment}

The authors wish to express their gratitude to all members of the Science \& Technology on Inertial Laboratory, and Fundamental Science on Novel Inertial Instrument \& Navigation System Technology Laboratory.

\section{References}

[1] TITTERTON D, WESTON J. Strapdown inertial navigation technology. 2nd ed. Reston, VA: American Institute of Aeronautics and Astronautics, 2004.

[2] SIGEL D A, WETTERGREEN D. Star tracker celestial localization system for a lunar rover. Proc. of the IEEE/RSJ Inter- national Conference on Intelligent Robots and Systems, 2007: $2851-2856$.

[3] CHEN H M, XIONG Z, QIAO L, et al. Application of CNSINS integrated navigation technology in high-altitude aircraft. Transducer \& Microsystem Technologies, 2008, 27(9): 4-6. (in Chinese)

[4] HONG D, LIU G B, CHEN H M, et al. Application of EKF for missile attitude estimation based on "SINS/CNS" integrated guidance system. Proc. of the International Symposium on Systems and Control in Aeronautics and Astronautics, 2010: $1101-1104$.

[5] WU X J, WANG X L. A SINS/CNS deep integrated navigation method based on mathematical horizon reference. Aircraft Engineering and Aerospace Technology, 2011, 83(1): 26-34.

[6] HE Z, WANG X L, FANG J C. An innovative high-precision SINS/CNS deep integrated navigation scheme for the Mars rover. Aerospace Science and Technology, 2014, 39: 559566.

[7] NING X L, LIU L L. A two-mode INS/CNS navigation method for lunar rovers. IEEE Trans. on Instrumentation and Measurement, 2014, 63(9): 2170-2179.

[8] WANG R, XIONG Z, LIU J Y, et al. A new tightly-coupled INS/CNS integrated navigation algorithm with weighted multi-stars observations. Proc. of the Institution of Mechanical Engineers Part G Journal of Aerospace Engineering, 2015, 230(4): $698-712$.

[9] NING X L, WANG F, FANG J C. Implicit UKF and its observability analysis of satellite stellar refraction navigation system. Aerospace Science \& Technology, 2016, 54: 49-58.

[10] GAO P Y, LI K, SONG T X, et al. An accelerometers size effect self-calibration method for tri-axis rotational inertial navigation system. IEEE Trans. on Industrial Electronics, 2017, 65(2): $1655-1664$.

[11] ZHENG Z C, HAN S L, YUE J, et al. Compensation for stochastic error of gyros in a dual-axis rotational inertial navigation system. Journal of Navigation, 2015, 69(1): 1-14.

[12] LV P, LIU J Y, LAI J Z, et al. Decrease in accuracy of a rotational SINS caused by its rotary table's errors. International Journal of Advanced Robotic Systems, 2014, 11(1): 1-10. 
[13] WANG B, REN Q, DENG Z Z, et al. A self-calibration method for nonorthogonal angles between gimbals of rotational inertial navigation system. IEEE Trans. on Industrial Electronics, 2015, 62(4): 2353-2362.

[14] WU W R, NING X L, LIU L L. New celestial assisted INS initial alignment method for lunar explorer. Journal of System Engineering and Electronics, 2013, 24(1): 108-117.

[15] SUN Y, WANG T J, GAO Y B, et al. Computation structure of rotating strapdown INS. Journal of Chinese Inertial Technology, 2013, 21(1): 10 - 15. (in Chinese)

[16] LI K, XU Y F, ZHANG Z Y, et al. Errors autocompensation principle analysis and experiments verification for rotational inertial navigation systems. Systems Engineering and Electronics, 2011, 33(10): 2268-2271. (in Chinese)

[17] LI K, GAO P Y, WANG L, et al. Analysis and improvement of attitude output accuracy in rotation inertial navigation system. Mathematical Problems in Engineering, 2015, 2015: 768174.

[18] SUN W, XU A G, CHE L N, et al. Accuracy improvement of SINS based on IMU rotational motion. IEEE Aerospace \& Electronic Systems Magazine, 2012, 27(8): 4-10.

[19] LV P, LAI J Z, LIU J Y, et al. Compensation effects of gyros' stochastic errors in a rotational inertial navigation system. Journal of Navigation, 2014, 67(6): 1069 - 1088.

[20] ZHOU Y, DENG Z H, WANG B, et al. Analytical method of the performance of the rotational ins based on the spatial accumulation of the inertial instrument biases. IFAC Proceedings Volumes, 2014, 47(3): 9649-9653.

[21] RUFINO G, ACCARDO D. Enhancement of the centroiding algorithm for star tracker measure refinement. Acta Astronautica, 2003, 53(2): $135-147$.

[22] YOON H, LIM Y, BANG H. New star-pattern identification using a correlation approach for spacecraft attitude determination. Journal of Spacecraft and Rockets, 2011, 48(1): $182-$ 186.

[23] NING X L, WANG L H, WU W R, et al. A celestial assisted INS initialization method for lunar explorers. Sensors, 2011, 11(7): $6991-7003$.

[24] JAFARZADEH S, LASCU C, FADALI M S. State estimation of induction motor drives using the unscented Kalman filter. IEEE Trans. on Industrial Electronics, 2012, 59(11): 4207 4216.

[25] JULIER S J, UHLMANN J K. Unscented filtering and nonlinear estimation. Proceedings of the IEEE, 2004, 92(3): $401-$ 422.

[26] HAVSTAD S A. Star sensor simulation for astroinertial guidance and navigation. Proc. of the SPIE, 1992: 74-84.

[27] WEI X G, ZHANG G J, FAN Q Y, et al. Star sensor calibration based on integrated modelling with intrinsic and extrinsic parameters. Measurement, 2014, 55: 117-125.

[28] ISHIBASHI S, TSUKIOKA S, YOSHIDA H, et al. Accuracy improvement of an inertial navigation system brought about by the rotational motion. Proc. of the Oceans, 2007: 1-5.

\section{Biographies}

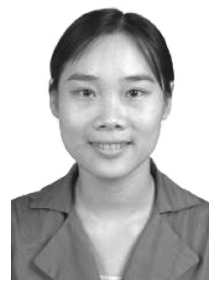

NING Xiaolin was born in 1979. She received her B.E. degree in computer science from Shandong Normal University, Shandong, China, in 2001, and $\mathrm{Ph} . \mathrm{D}$. degree in mechanical engineering from Beihang University, Beijing, China, in 2008. She has been a professor with the School of Instrument and Optoelectronic Engineering, Beihang University since 2011. From March 2014 to March 2015, she was a visiting scholar at the National University of Singapore. Her research interests include guidance, navigation, and control system of spacecraft and autonomous navigation of deep space explorers. She proposed many novel methods of astronomical navigation, and the research results are used in the Chang'e II and Mars exploration pre-research.

E-mail: ningxiaolin@buaa.edu.cn

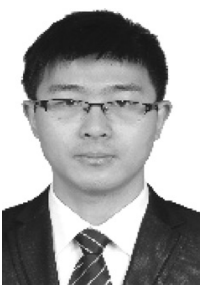

YUAN Weiping was born in 1992. He received his B.E. degree in measurement and control technology and instrument from University of Science and Technology, Beijing, in 2015. He received his master degree from Beihang University in 2018. Now, he is engaged in navigation related job as a engineer at Huawei, Beijing. His research interests include single-axis rotational SINS/CNS integrated navigation, vehicle navigation and driver-less car. E-mail: yuan_1166@sina.com

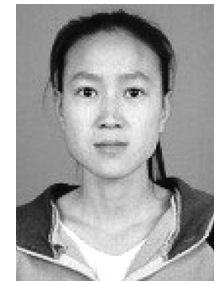

LIU Yanhong was born in 1988. She received her B.E. degree in electrical engineering and automation from Hebei University of Engineering in 2014. She received her master degree in control science and engineering from Beijing University of Technology in 2017. Since 2017, she has been working toward her doctor degree in Beihang University. In the first year, she served as a teaching assistant in the School of Instrument and Optoelectronic Engineering. Her research interests include machine vision, indoor navigation and high precision strapdown inertial navigation system/global positioning system integrated measurement system.

E-mail: liu1253891321@163.com 